\title{
Routinemäßige Ganzhirnbestrahlung ist nicht gerechtfertigt
}

Hintergrund und Fragestellung: Kann bei Patienten mit multiplen Hirnmetastasen nichtkleinzelliger Lungenkarzinome (NSCLC) auf die Ganzhirnbestrahlung verzichtet werden? Hirnmetastasen sind beim NSCLC sehr häufig und stellen aufgrund der hohen Inzidenz dieses Tumors in Europa eine große Herausforderung dar. Wenn lokale Therapiemaßnahmen wie Resektion oder stereotaktische Radiotherapie nicht sinnvoll erscheinen, wird in der Regel eine Ganzhirnbestrahlung durchgeführt. Deren Stellenwert im Hinblick auf eine Verbesserung der Lebensqualität und einer Lebenszeitverlängerung wurde in der QUARTZ(Quality of Life after Treatment for Brain Metastases)-Studie auf den Prüfstand gestellt.

\section{Originalie}

Mulvenna P et al. Dexamethasone and supportive care with or without whole brain radiotherapy in treating patients with non-small cell lung cancer with brain metastases unsuitable for resection or stereotactic radiotherapy (QUARTZ): results from a phase 3 , non-inferiority, randomised trial. Lancet. 2016; 388(10055):2004-14.
Patienten und Methodik: Die Nichtunterlegenheitsstudie der Phase III wurde an 69 britischen und drei australischen Zentren durchgeführt. NSCLC-Patienten mit Hirnmetastasen erhielten randomisiert entweder eine optimale supportive Behandlung (optimal supportive care, OSC) einschließlich Dexamethason und Ganzhirnbestrahlung $(5 \times 4 \mathrm{~Gy})$, oder OSC allein. Die Dexa-
methason-Dosis wurde den behandelnden Teams überlassen. Der primäre Endpunkt der Studie waren „Quality-adjusted life years" (QALY), basierend auf dem Gesamtüberleben und wöchentlichem Einsatz des EQ-5D-Fragebogens. Die Auswertung wurde nach dem Intention-to-treat-Prinzip (ITT) für alle randomisierten Patienten durchgeführt.

Ergebnisse: Von März 2007 bis August 2014 wurden 538 Patienten rekrutiert. Das mediane Alter betrug 66 Jahre (Bereich 38-85 Jahre). Die Patienten im Strahlentherapiearm erlitten häufiger Haarausfall, Übelkeit oder Hautsymptome, aber die Inzidenz ernster Nebenwirkungen war in beiden Studienarmen vergleichbar. Es gab keinen Unterschied in der Gesamtüberlebenszeit zwischen Patienten ohne versus mit Strahlentherapie (Hazard Ratio 1,06, 95\%-Konfidenzintervall 0,9-1,26). Auch zeigten sich keine Unterschiede im Hinblick auf die Lebensqualitätsdaten oder den Dexamethason-Gebrauch. Der Unterschied zwischen den QALY betrug 4,7 Tage: 46,4 Tage für OSC plus Ganzhirnbestrahlung versus 41,7 Tage für OSC allein.

Schlussfolgerungen der Autoren: Die Ganzhirnbestrahlung bringt für betroffene Patienten gemäß Einschlusskriterien der Studie allenfalls einen geringen klinischen Gewinn, der ihren routinemäßigen Einsatz nicht rechtfertigt.

Schlüsselwörter: NSCLC - Hirnmetastasen - Ganzhirnbestrahlung - Lebensqualität

\section{- Kommentar von Michael Weller, Zürich, Schweiz}

\section{„Auch etablierte Therapiekonzepte müssen überprüft werden"}

QUARTZ ist eine eindrucksvolle Studie, deren erfolgreiche Durchführung über Jahre bezweifelt wurde, weil die Randomisierung der Patienten zur Überprüfung der Fragestellung ethisch problematisch erschien. Sie ist ein eindrucksvolles Beispiel dafür, dass es sinnvoll sein kann, auch über Dekaden etablierte Therapieformen einer kritischen Prüfung zu unterziehen. In der Diskussion gehen die Autoren sorgfältig mit den Ergebnissen ihrer Subgruppenanalysen um und weisen darauf hin, dass für jüngere Patienten in besserem Allgemeinzustand möglicherweise ein größerer Zugewinn durch die Bestrahlung besteht, der in dieser Studie nicht abschließend beurteilt werden kann.

Es war auch interessant zu beobachten, dass zumindest in einer diesbezüglich unkontrollierten Studie keine größere Reduktion der Kortisondosen erfolgte, wenn die Patienten bestrahlt wurden. Zu berücksichtigen ist auch, dass $11 \%$ von den der Ganzhirnbestrahlung zugeordneten Patienten diese Therapie nicht erhielten. Mit dem zunehmenden Einsatz der stereotaktischen Radiotherapie und der zunehmenden Verfüg- barkeit zielgerichteter pharmakologischer Therapien, die über einen größeren Zeitraum der vorliegenden Studie noch nicht verfügbar waren, wird sich die Therapie von NSCLC-Patienten mit Hirnmetastasen weiter von der Ganzhirnbestrahlung entfernen, sobald diese wichtigen Studienergebnisse von einer größeren Gruppe zuständiger Ärzte weltweit zur Kenntnis genommen worden sind.

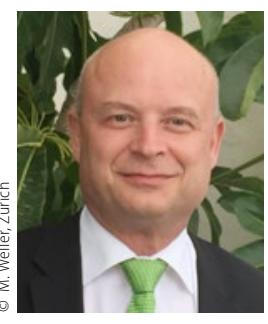

Prof. Dr. Michael Weller

Direktor der Klinik für Neurologie, Universitätsspital Zürich, Schweiz michael.weller@usz.ch 